\title{
A Young man with Peri-ictal water drinking
}

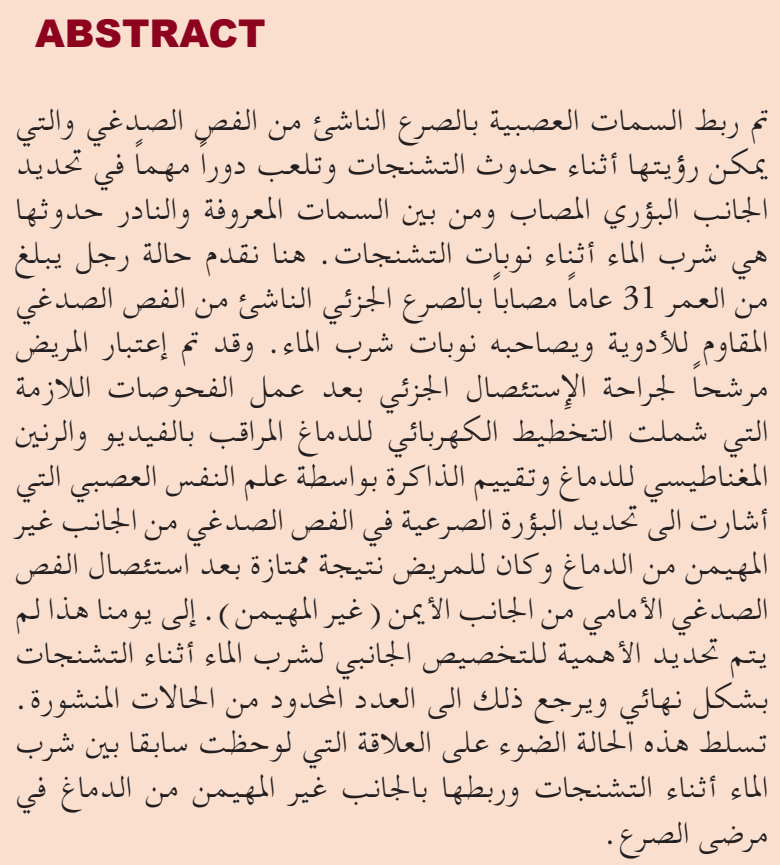

Neuro-vegetative features have been linked to epilepsy arising from the temporal lobe, which can be seen during ictal events and play an important role in determining the focal side of the lesion. Among the rare known features is peri-ictal water drinking (PIWD). Here, we present the case of a 31-year-old male with refractory temporal epilepsy, having episodes of PIWD. The patient was considered a candidate for resective surgery and was investigated accordingly, including long-term video electroencephalogram (EEG), brain magnetic resonance imaging (MRI), and neuropsychology assessment, which pointed towards a lesioned temporal lobe over the nondominant hemisphere. The patient had an excellent outcome following right anterior temporal lobectomy. The lateralization significance of PIWD has not yet been established definitively in the literature, partly due to a limited number of published cases. This case highlights the previously observed association between PIWD and the involvement of the nondominant hemisphere in epilepsy patients.

Neurosciences 2021; Vol. 26 (1): 85-88 doi: 10.17712/nsj.2021.1.20200074
From the Department of Pediatric Neurology, Epilepsy Monitoring Unit, National Neuroscience Institute, King Fahad Medical City, Riyadh, Kingdom Of Saudi Arabia.

Received 12th May 2020. Accepted 6th August 2020.

Address correspondence and reprint request to: Dr. Ghalib M. Alanazi, Department of Pediatric Neurology, Epilepsy Monitoring Unit, National Neuroscience Institute, King Fahad Medical City, Riyadh, Kingdom of Saudi Arabia. E-mail: ghalib-444@hotmail.com ORCID ID: https://orcid.org/0000-0002-7474-5473

$\mathrm{T}$ he initial description of peri-ictal water drinking (PIWD) dates back to the early twentieth century with observations by William G. Lennox and Stanley Cobb. ${ }^{1}$ However, drinking water is a usual habitual action, so it is difficult for patients or witnesses to consider this behavior as part of ictal manifestation, which made PIWD largely underestimated. Neurovegetative features such as mydriasis, transient hypo- or hypertension, and an increase in heart rate are well known and occur quite frequently in temporal lobe seizures. Many other features are described and occur much less frequently, as is the case in PIWD. ${ }^{2}$ Review of the literature showed only a handful of studies looking into PIWD, and no definite evidence exists to answer all questions about its relevance to temporal lobe epilepsy (TLE); however, a possible lateralizing value has been hypothesized. ${ }^{3}$ We present a case of right symptomatic TLE secondary to mesial temporal sclerosis (MTS) with PIWD behavior episodes confirmed by ictal video-electroencephalogram (EEG) that was recorded in an epilepsy monitoring unit. We also reviewed the literature to evaluate the localization and lateralization significance of this manifestation in TLE.

Disclosure. The authors declare no conflicting interests, support or funding from any drug company. 


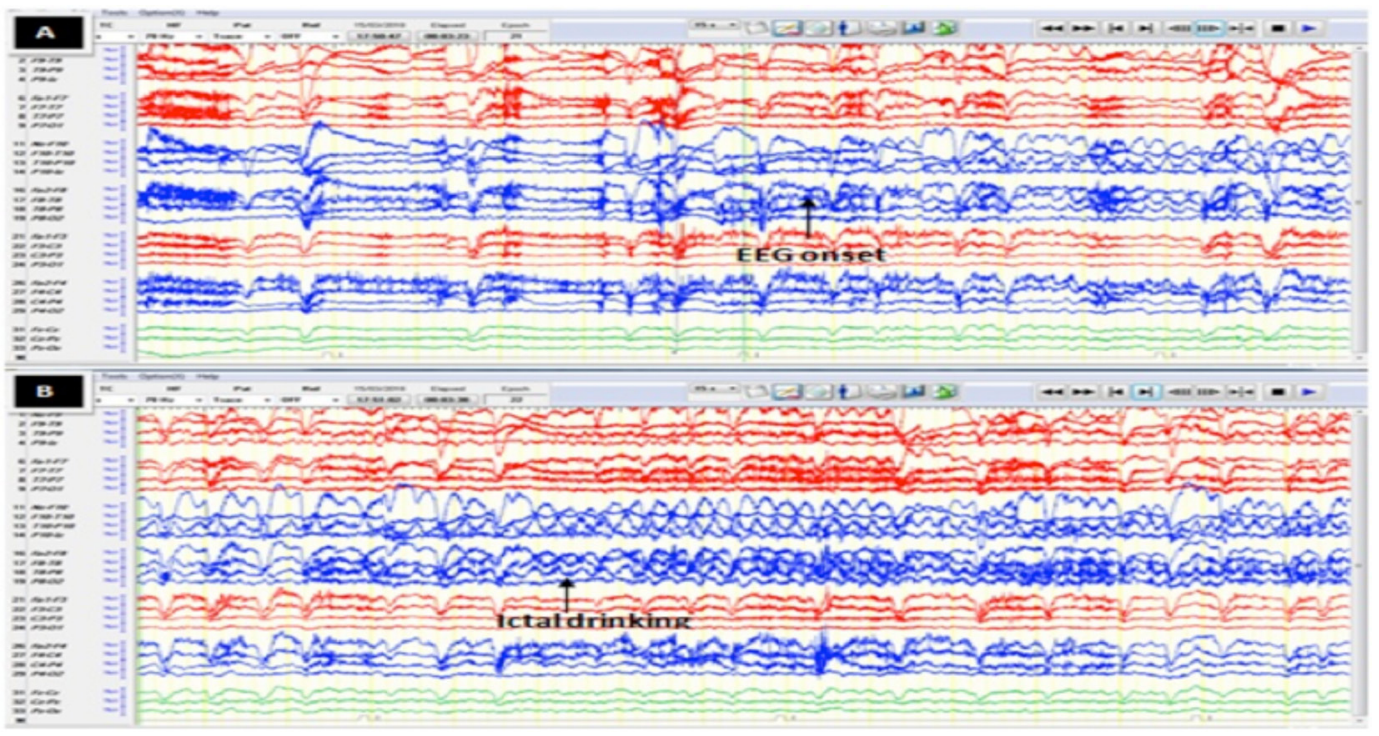

Figure 1 - Electroencephalogram (EEG) (A) Ictal EEG onset with rhythmic discharges started from the right temporal region; (B) High amplitude rhythmic discharges in the right temporal region (along with the beginning of ictal water drinking 30 seconds after EEG onset).
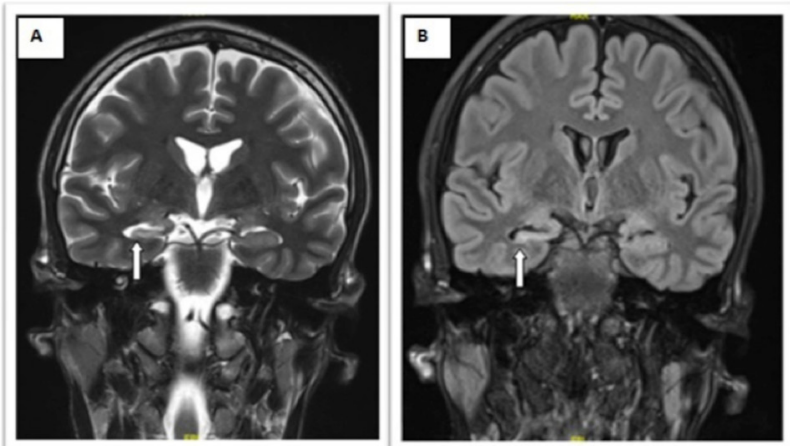

Figure 2 - The coronal T2WI (A) and FLAIR (B) images showing right-sided mesial temporal sclerosis. Notice the increased signal and small hippocampus with loss of normal internal architecture of the right hippocampus and dilated right temporal horn.

\section{Case Report. Patient information and clinical} findings. A 31-year-old left-handed male known to have epilepsy since the age of 7 years presented to our epilepsy center at King Fahad Medical City, Riyadh, Kingdom of Saudi Arabia. His seizures were characterized by palpitations and thirst followed by right-hand automatism. He reported partial intact awareness and orientation at first, but later he could not recall what had happened. No other seizure types or history of febrile convulsion were documented. The treatment regimen consisted of valproic acid and levetiracetam (1 $\mathrm{g}$ bid for each drug) with no seizure control. Clinical and neurological examinations were unremarkable.
Diagnostic assessment. The patient was admitted to our epilepsy center for preoperative evaluation, including video-EEG monitoring, brain magnetic resonance imaging (MRI), positron emission tomography (PET) scanning, and a neuropsychological assessment. The patient had 6 seizures during hospitalization secondary to the gradual discontinuation of antiepileptic drugs (AEDs). The episodes were characterized by sudden mild headaches, palpitations, and excessive drinking of water followed by left-hand dystonic contraction. During these episodes, he was able to speak and gave meaningful answers to questions; later, he became disoriented. Ictal EEG changes began $30 \mathrm{sec}$ before clinical onset and showed a buildup of rhythmic theta activity over the right anterior and mid-temporal head regions (Figure 1A, B). No EEG changes were noted post-ictally. Brain MRI showed right mesial temporal sclerosis (Figure 2A, B), and a PET scan revealed hypometabolism in the right temporal lobe. Neuropsychological evaluation results were within normal limits.

Therapeutic intervention, follow-up, and outcome. The patient underwent a right anterior temporal resection. He was seizure-free at the one-year follow-up; however, he remained on AEDs. His follow-up EEG showed residual epileptic activity (Figure 3).

Discussion. The PIWD is an uncommon feature described in patients with lesional and non-lesional epilepsy of the temporal lobe. It is defined as a stereotyped action seen across all age groups during 


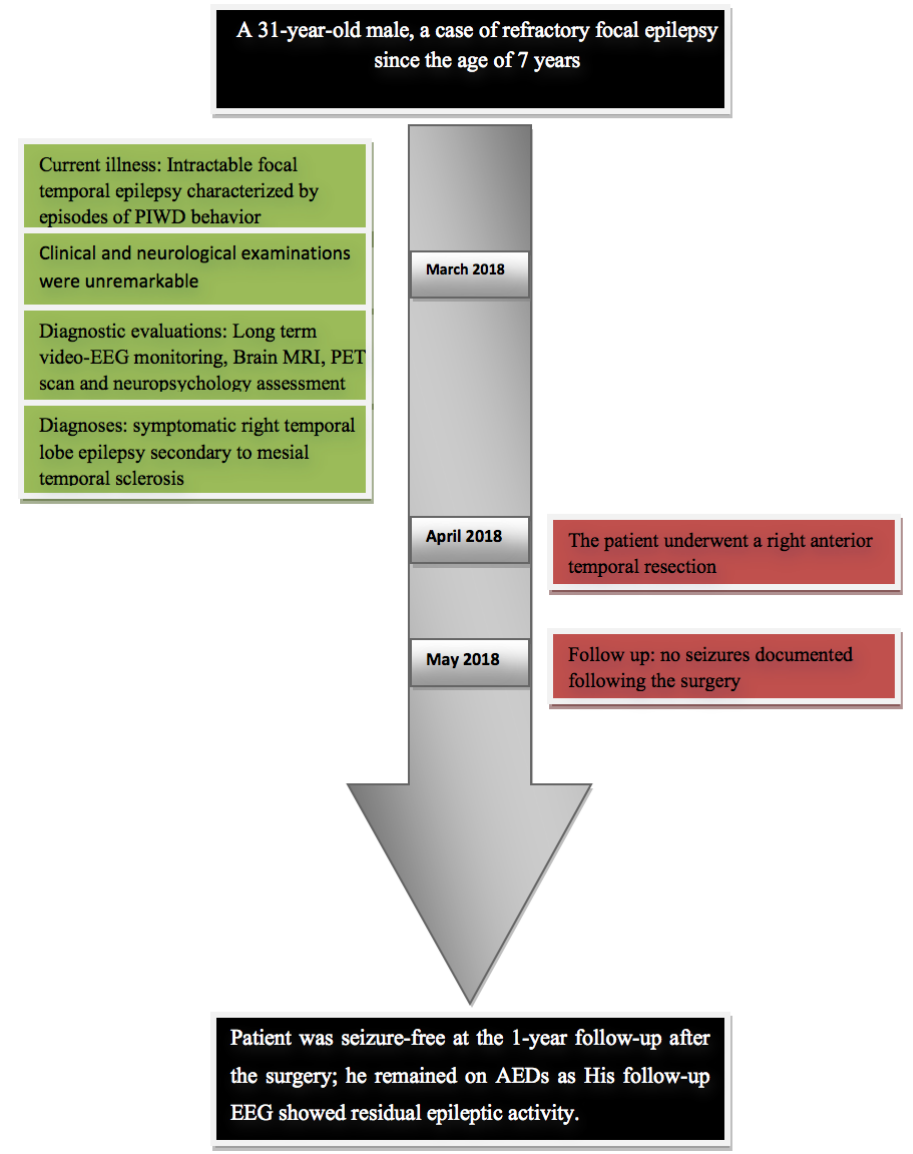

Figure 3 - Time line figure of the presented case.

seizures or within 1 to 2 minutes of their onset. ${ }^{3}$ The described case in this report concerned lesional epilepsy of the temporal lobe as documented in neuroimaging over the right side with automatic PIWD during video-EEG documented seizures, confirming the ictal nature of this manifestation.

To date, 64 cases of epileptic seizures associated with PIWD have been reported. ${ }^{4}$ The rate of reported cases of PIWD seen in TLE ranged from $7 \%$ to $15.3 \%$. $^{2,3,5,6}$ It was observed in $15.3 \%$ of 386 seizures in 65 patients with focal epilepsy reported by Trinka et $\mathrm{al}^{3}$. In a study of 55 adult patients with TLE, PIWD was seen in 8 patients $(14.5 \%) .^{6}$ Janszky et $\mathrm{al}^{5}$ observed PIWD in $7 \%$ of 141 adult patients with TLE. Musilová et $\mathrm{al}^{2}$ reported PIWD in $14.4 \%$ of 380 seizures in 97 patients with TLE-associated vegetative signs.

Generally, favorable surgical outcomes have been described for TLE patients with PIWD. A case series published by Trinka et al. ${ }^{3}$ demonstrated a complete response to surgical treatment in $70 \%$ of the studied cohort (Wieser's class I). Similar favorable results of surgery were seen with a group of patients studied by Szücs et al. ${ }^{6}$ (Engel Class 1 or 2) with the outcome maintained for a few years of postoperative follow-up. Our patient was seizure-free at the one-year follow-up after surgery.

Normal drinking behavior is controlled by vascular volume and tissue osmolality. Information from peripheral receptors is processed in the hypothalamus and then conveyed to various brain structures that initiate a search for water and drink. ${ }^{7}$ Furthermore, the hippocampus and the amygdala are linked to the hypothalamus through several multi-synaptic pathways that are involved in the regulation of water balance and drinking behavior. ${ }^{8}$ Water-seeking behavior is triggered by the involvement of the hypothalamus, which is affected by abnormal electrical activity generated by the temporal lobe. ${ }^{9}$

The PIWD is thought to reflect involvement of the non-dominant hemisphere because neural networks 
involved in fluid balance, thirst, and water-seeking are normally asymmetrical and are likely more active in the non-dominant side. ${ }^{10}$ The lateralizing value of PIWD remains incompletely determined. Some studies have linked PIWD to significant lateralization to the nondominant hemisphere in patients with TLE. ${ }^{2,3}$ Other reports could not demonstrate clear evidence for the lateralization value of PIWD. ${ }^{5,6}$ This uncertainty could be due to the rapid spread of epileptic discharges from the focal source of the seizures to the contralateral side of the temporal lobe, or it may be due simply to the few reported cases of PIWD. ${ }^{6}$

In conclusion, PIWD is a rare and important vegetative symptom of TLE that can be a valuable lateralizing sign to the non-dominant hemisphere. We report here a case of PIWD as a manifestation of TLE in the non-dominant hemisphere; however, the association of PIWD with a non-dominant hemisphere has not yet been definitively established in the literature. Given the significance of PIWD in planning surgical resection, a more comprehensive and powerful study with enrollment of more patients is needed. Furthermore, the lateralization could be supported and confirmed by invasive EEG recordings.

Acknowledgements. We would like to thank Dr. Alawi Al-Attas and Dr. Majed Alhameed for their kind support, guidance, and motivation to report this case. Also, I would like to thanks Dr. Eman Bakhsh, neuroradiologist consultant for reviewing the images of the patient, and Scribendi (www.scribendi.com) for English language editing.

\section{References}

1. Lennox WG, Cobb S. Epilepsy: XIII. Aura IN Epilepsy; A Statistical Review OF 1,359 Cases. Arch NeurPsych 1933; 30: 374-387.

2. Musilová K, Kuba R, Brázdil M, Tyrlíková I, Rektor I. Occurrence and lateralizing value of "rare" peri-ictal vegetative symptoms in temporal lobe epilepsy. Epilepsy Behav 2010; 19: 372-375.

3. Trinka E, Walser G, Unterberger I, Luef G, Benke T, Bartha $\mathrm{L}$, et al. Peri-ictal water drinking lateralizes seizure onset to the nondominant temporal lobe. Neurology 2003; 60: 873-876.

4. Huang S, Al-Abri H, Sachdeva A, Alkhachroum AM, Shatzman $S$, Lüders H. Recurrent focal seizures as a feature of status epilepticus presenting as a peri-ictal water drinking. Epilepsy Behav Case Rep 2018; 10: 129-132.

5. Janszky J, Fogarasi A, Toth V, Magalova V, Gyimesi C, Kovacs $\mathrm{N}$, et al. Peri-ictal vegetative symptoms in temporal lobe epilepsy. Epilepsy Behav 2007; 11: 125-129.

6. Szucs A, Fogarasi A, Rásonyi G, Kelemen A, Narula L, Tóth V, et al. Peri-ictal water drinking in temporal lobe epilepsy: Is it a reliable lateralizing sign? Epilepsy Behav 2007; 11: 578-781.

7. Denton DA, McKinley MJ, Weisinger RS. Hypothalamic integration of body fluid regulation. Proceedings of the National Academy of Sciences 1996; 93: 7397-7404.

8. Gloor P, Guberman AH. The temporal lobe \& limbic system. Can Med Assoc J 1997; 157: 1597.

9. Sibole W, Miller JJ, Mogenson GJ. Effects of septal stimulation on drinking elicited by electrical stimulation of the lateral hypothalamus. Exp Neurol 1971; 32: 466-477.

10. Denton D, Shade R, Zamarippa F, Egan G, Blair-West J, McKinley M, et al. Correlation of regional cerebral blood flow and change of plasma sodium concentration during genesis and satiation of thirst. Proc Natl Acad Sci U S A 1999; 96: 2532-2537.

\section{Case Reports}

Case reports will only be considered for unusual topics that add something new to the literature. All Case Reports should include at least one figure. Written informed consent for publication must accompany any photograph in which the subject can be identified. Figures should be submitted with a 300 dpi resolution when submitting electronically. The abstract should be unstructured, and the introductory section should always include the objective and reason why the author is presenting this particular case. References should be up to date, preferably not exceeding 15 . 